\title{
VEGF-C inhibition reverses resistance of bladder cancer cells to cisplatin via upregulating maspin
}

\author{
HAIPENG ZHU, FENG YUN, XIAOXUE SHI and DONG WANG \\ Department of Urinary Surgery, The Fifth Affiliated Hospital of Zhengzhou University, \\ Zhengzhou, Henan 450000, P.R. China
}

Received July 15, 2014; Accepted March 24, 2015

DOI: $10.3892 / \mathrm{mmr} .2015 .3684$

\begin{abstract}
The aim of the current study was to elucidate the association between vascular endothelial growth factor C (VEGF-C) and resistance of bladder cancer cells to cisplatin and the underlying mechanism involving maspin. A total of 32 bladder cancer tissue samples from patients (18 males and 14 females with an average age of 65.9 years) were collected from the Fifth Affiliated Hospital of Zhengzhou University (Zhengzhou, China). All patients had undergone cisplatin-based combination chemotherapy. In addition, the BIU87 human bladder cancer cell line was cultured and a cisplatin-resistant subline (BIU87-CisR) was established by continuous exposure to cisplatin. The mRNA expression levels of VEGF-C and maspin in tissue samples, BIU87 cells and BIU87-CisR cells were analyzed by reverse transcription-quantitative polymerase chain reaction ( $\mathrm{RT}-\mathrm{qPCR}$ ). Targeted inhibition of VEGF-C in BIU87-CisR cells was performed using small interfering (si)RNA technology and the alteration in levels of maspin was confirmed by RT-qPCR and western blot analysis. siRNA-treated and -untreated BIU87-CisR cells were divided into the following four groups: Control group (no drug treatment), $3 \mu \mathrm{M}$ cisplatin treated group, $3 \mu \mathrm{M}$ cisplatin + siRNA treated group and the siRNA treated group. Cell viability following treatment in each group was evaluated by the cell counting kit 8 assay. The cell cycle and apoptotic rate of BIU87-CisR cells was analyzed by propidium iodide (PI) staining and Annexin V-PI double staining with flow cytometry. Furthermore, pcDNA-maspin transfected BIU78-CisR cells were used to establish the effect of maspin on the sensitivity to cisplatin. VEGF-C expression in chemoresistant patients and BIU87-CisR cells was significantly increased compared with chemosensitive patients and normal BIU87
\end{abstract}

Correspondence to: Mr. Haipeng Zhu, Department of Urinary Surgery, The Fifth Affiliated Hospital of Zhengzhou University, 3 Kangfuqian, Zhengzhou, Henan 450000, P.R. China

E-mail: zhuhaipeng_surgey@163.com

Key words: vascular endothelial growth factor-C, bladder cancer, cisplantin, maspin cells, respectively. By contrast, maspin levels were lower in chemoresistant patients and BIU87-CisR cells. Subsequent to VEGF-C inhibition, maspin expression was markedly increased. Cisplatin $(3 \mu \mathrm{M})$ resulted in moderate proliferation inhibition of BIU87-CisR cells without siRNA pretreatment; however, significant inhibition was observed in the VEGF-C siRNA treated group. In addition, the cell cycle arrest and apoptosis induced by cisplatin was enhanced by VEGF-C inhibition. Overexpression of maspin was able to improve the sensitivity of BIU87-CisR cells to cisplatin. In conclusion, the resistance of bladder cancer cells to cisplatin may be induced by upregulation of VEGF-C, and inhibition of VEGF-C reverses resistance by elevating maspin expression levels.

\section{Introduction}

In China, bladder cancer has the highest rate of incidence out of all malignancies of the urinary system (1). In addition to surgical treatment, chemotherapy is an important strategy for the therapy of bladder cancer. Cisplatin-based chemotherapy is widely used in bladder cancer treatment, and the anti-cancer effect was demonstrated (2). However, certain patients exhibit a poor sensitivity to cisplatin, and this resistance to cisplatin is a problem that should not be overlooked. The low sensitivity to cisplatin and drug resistance affect the therapeutic efficacy of bladder cancer treatment $(3,4)$. Therefore, it is necessary to investigate the mechanism of resistance of bladder cancer to cisplatin and aim to improve the sensitivity of bladder cancer cells to this drug.

Vascular endothelial growth factor C (VEGF-C) is a dimeric glycoprotein of the VEGF family of cytokines. VEGF-C has been demonstrated to be involved with the majority of aggressive tumors (5). A number of previous studies have reported that high levels of VEGF-C promote tumor invasion and metastasis by binding to its receptor $(6,7)$. Clinical studies have verified that VEGF-C expression is closely associated with the invasive phenotype and affects patient survival in cervical cancer, in addition to accelerating cervical cancer metastasis by directly driving cancer cell migration and invasion (8). In brief, high levels of VEGF-C correlate with poor prognosis for the patient (8). However, few studies investigating whether high expression levels of VEGF-C are implicated in chemoresistance have been conducted. One previous study suggested that the overexpres- 
sion of VEGF-C induced chemoresistance in acute myeloid leukemic cells via a cyclooxygenase-2-mediated mechanism. Cho et al (9) demonstrated that RhoGDI2-induced VEGF-C expression results in gastric cancer cell metastasis and cisplatin resistance. Therefore, based on the previous studies, it was hypothesized that high expression levels of VEGF-C result in chemoresistance to cisplatin in bladder cancer cells. Furthermore, it was hypothesized that maspin may mediate the effects of VEGF-C in regulating chemoresistance. As an inhibitor of serine protease, accumulating evidence indicates that maspin is able to inhibit the growth of tumors by inducing apoptosis (10). In certain types of tumor, low expression of maspin may induce growth of tumours (11). Induction of apoptosis is a crucial function of chemotherapeutic drugs, therefore, it is important to analyze the association between maspin and the effectiveness of chemotherapy. In a previous study, the elevated expression level of maspin was observed to be typical for cisplatin-sensitive ovarian cancer tumors (12). Thus, it was considered that maspin is associated with the sensitivity of bladder cancer cells to cisplatin. The current study utilized small interfering (si)RNA technology to inhibit VEGF-C expression in BIU87-CisR cells, then observed the alterations in sensitivity to cisplatin of BIU87-CisR cells, and the alterations in maspin levels following VEGF-C inhibition.

\section{Materials and methods}

Patients and preparation for tissue specimens. The current study included 32 patients with bladder cancer (18 males and 14 females; median age, 65.9; range 51-76) who underwent surgical treatment and cisplatin-based combination chemotherapy between March 2012 and February 2013 at the Fifth Affiliated Hospital of Zhengzhou University (Zhengzhou, China). A total of 20 patients were sensitive to chemotherapy, while 12 were resistant to it, while no significant correlations were observed with regard to the demographic information about age, gender, stage of disease and treatment regimen. Tumor tissue specimens were obtained at the time of surgical esophageal tissue resection and stored in liquid nitrogen until further analysis. The current study was approved by the ethics committee of The Fifth Affiliated Hospital of Zhengzhou University, with all patients' informed consents.

Cell line culture and establishment of cisplatin-resistant subline. The BIU87 cell line was purchased from the Type Culture Collection of the Chinese Academy of Sciences (Shanghai, China). BIU87 cells were maintained in RPMI-1640 medium (Thermo Fisher Scientific, Waltham, MA, USA) supplemented with $10 \%$ fetal bovine serum at $37^{\circ} \mathrm{C}$ with $5 \%$ $\mathrm{CO}_{2}$. The cisplatin-resistant subline (BIU87-CisR cell line) was established as previously described and its resistance to cisplatin (Sigma-Aldrich, St. Louis, MO, USA) was proven (13). In brief, BIU87-CisR cells were obtained from parental BIU87 cells through a continuous exposure to increasing cisplatin over 12 months, with a final concentration of $6 \mu \mathrm{M}$ cisplatin.

RNA isolation and quantification of VEGF-C mRNA expression. Reverse transcription-quantitative polymerase chain reaction (RT-qPCR) was performed to quantify the mRNA expression of VEGF-C. Total RNA of tumor tissue specimens,
BIU87 cells and BIU87-CisR cells were extracted using TRIzol reagent (Thermo Fisher Scientific). The purity and concentration of total RNA was verified by spectrophotometry (Biomate 3; Thermo Fisher Scientific). Confirmed RNA was reverse-transcribed to cDNA using PrimeScript ${ }^{\circledR}$ RT reagent (Takara Biotechnology Co., Ltd., Dalian, China) according to manufacturer's instructions. cDNA was then amplified with SYBR ${ }^{\circledR}$ Premix Ex Taq ${ }^{\mathrm{TM}}$ II kit (Takara Biotechnology Co., Ltd.) using a 7500 Real Time PCR system (Applied Biosystems Life Technologies, Foster City, CA, USA). The cycling conditions were set according to the manufacturer's instructions. The sequences of the primers of targeted genes and $\beta$-actin used were as follows: Forward: 5'CAAGCATGGCCTGTA CAACCTC'3 and reverse: 5'GGGTTCACACACCAGCAC TC'3 for VEGF-C; and forward: 5'ATCATGTTTGAGACC TTCAA' and reverse: 5'CATCTCTTGCTCGAAGTCCA'3 $\beta$-actin. The fold changes of target genes were calculated using the $\Delta \Delta$ cycle threshold $\left(2^{-\Delta \Delta \mathrm{Ct}}\right)$ method and the result was normalized to $\beta$-actin.

Western blot analysis for VEGF-C protein expression. Protein samples of tissue, BIU87 cells and BIU87-CisR cells were prepared using radioimmunoprecipitation assay buffer combined with $1 \%$ protease inhibitor cocktail (Applygen Technologies, Inc., Beijing, China). Protein samples (30 $\mu \mathrm{g})$ were separated by $12 \%$ SDS-PAGE (100 V, $1.5 \mathrm{~h})$ and transferred to polyvinylidene difluoride membranes $(150 \mathrm{~mA}, 1 \mathrm{~h}$; Applygen Technologies, Inc.). Membranes were blocked with $5 \%$ skimmed milk in phosphate-buffered saline (PBS) for $1 \mathrm{~h}$ and incubated at $4^{\circ} \mathrm{C}$ for $12 \mathrm{~h}$ with the primary antibody mous anti-VEGF-C monoclonal (1:1,000; cat. no. sc-374628; Santa Cruz Biotechnology, Inc., Dallas, TX, USA) and mouse anti- $\beta$-actin monolonal (1:5,000; cat. no. sc-130300; Santa Cruz Biotechnology, Inc.). Membranes were washed by PBS with Tween 20 buffer and followed an incubation with horseradish peroxidase-conjugated secondary antibodies. The bands were detected using an Enhanced Chemiluminescence Detection Reagent kit (Applygen Technologies, Inc.) and analyzed by Image J software version 1.48 (NIH, Bethesda, MD, USA).

Transfection of VEGF-C siRNA. Downregulation of VEGF-C expression in BIU87-CisR cells was induced using siRNA. siRNA targeted to human VEGF-C were designed and synthesized by Shanghai GenePharma Co., Ltd. (Shanghai, China) and then transfected into cells using Lipofectamine ${ }^{\circledR} 2000$ reagent (Life Technologies, Grand Island, NY, USA). The concentration of siRNA was $3 \mathrm{nM}$ and BIU87-CisR cells were cultured $48 \mathrm{~h}$ following transfection. The silencing effect was assessed at the mRNA and protein expression levels in the preliminary experiment and the effect of siRNA transefection was efficient.

Overexpression of maspin. The pcDNA-maspin recombinant plasmids were constructed (Data not shown) and were transfected into BIU87-CISR cells using Lipofectamine 2000 reagent in order to increase the expression of maspin.

Cell inhibition analysis. Cell inhibition induced by cisplatin was detected by cell counting kit 8 (CCK-8) kits (Wuhan Boster Biological Technology, Ltd., Wuhan, China). Normal BIU87 
cells and VEGF-C silenced BIU87-CisR cells were plated at a density of $1 \times 10^{4}$ cells/well in a 96 -well plate and divided into the following four groups: Control group, BIU87-CisR cells without any treatment; $3 \mu \mathrm{M}$ cisplatin-treated group, BIU87-CisR cells treated with $3 \mu \mathrm{M}$ cisplatin; $3 \mu \mathrm{M}$ cisplatin + siRNA treated group, VEGF-C silenced BIU87-CisR cells treated with $3 \mu \mathrm{M}$ cisplatin; and the siRNA group, VEGF-C silenced BIU87-CisR cells without cisplatin treatment. Following 24 h, $10 \mu$ l CCK-8 reagent was added and the cells were incubated at $37^{\circ} \mathrm{C}$ for $4 \mathrm{~h}$, then the optical density of the culture solution in each plate was measured using a Synergy Mx Microplate reader (BioTek Instruments, Inc., Winooski, VT, USA) at $450 \mathrm{~nm}$.

Cell cycle and cell apoptosis analysis. Cell cycle and cell apoptosis analysis was performed using flow cytometery. For cell cycle analysis, BIU87-CisR cells were collected and fixed with pre-cooled $70 \%$ ethanol. Subsequent to fixing for $12 \mathrm{~h}$, $500 \mu 1$ propidium iodide (Sigma-Aldrich, St. Louis, MO, USA) was added and cells were incubated for $30 \mathrm{~min}$. Cell cycle analysis was performed using a BD FACSCalibur flow cytometer (Beckman Coulter, Brea, CA, USA) for 15 min. For cell apoptosis analysis, the Annexin V-fluorescein isothiocyanate assay kit (Sigma-Aldrich) was used according to the manufacturer's instructions.

Analysis of maspin expression and its effect on the sensitivity of BIU87-CisR cells to cisplatin. Maspin expression in normal and VEGF-C silenced BIU87-CisR cells was detected by RT-qPCR and western blot analysis according to the above mentioned protocols. The sequences for the primers for maspin were as follows: Forward: 5'AACTGA AGATGGTGGGGATT'3 and reverse: 5'TGGGAAGAAGAG CTTCCAAA'3. Futhermore, the proliferation inhibition of maspin-overexpressing BIU87-CisR cells treated with $3 \mu \mathrm{M}$ cisplatin was analyzed using the CCK-8 kit.

Statistical analysis. All data are expressed as the mean \pm standard deviation. All calculations were performed using SPSS software, version 18.0 (SPSS, Inc., Chicago, IL, USA). A one-way analysis of variance followed by a least significant difference test was used to determine the statistical significance among four groups and between each group. $\mathrm{P}<0.05$ was considered to indicate a statistically significant difference.

\section{Results}

High VEGF-C expression levels were detected in the tumor tissue of chemotherapy-resistant patients and BIU87-CisR cells. As presented in Fig. 1, the mRNA levels of VEGF-C were higher $(1.6 \pm 0.03$ fold $)$ in chemoresistant patients $(n=20)$, compared with chemosensitive patients $(\mathrm{n}=12, \mathrm{P}<0.05)$. In addition, a higher (1.7 \pm 0.06 fold) mRNA level of VEGF-C was observed in BIU87-CisR cells (Fig. 2), compared with the parental BIU87 cells $(\mathrm{P}<0.05)$.

Western blot analysis confirmed the higher protein expression levels of VEGF-C in chemoresistant patients and BIU87-CisR cells (Figs. 3 and 4).

Knock-down of VEGF-C may enhance the proliferation inhibition effect of cisplatin. As presented in Fig. 5,

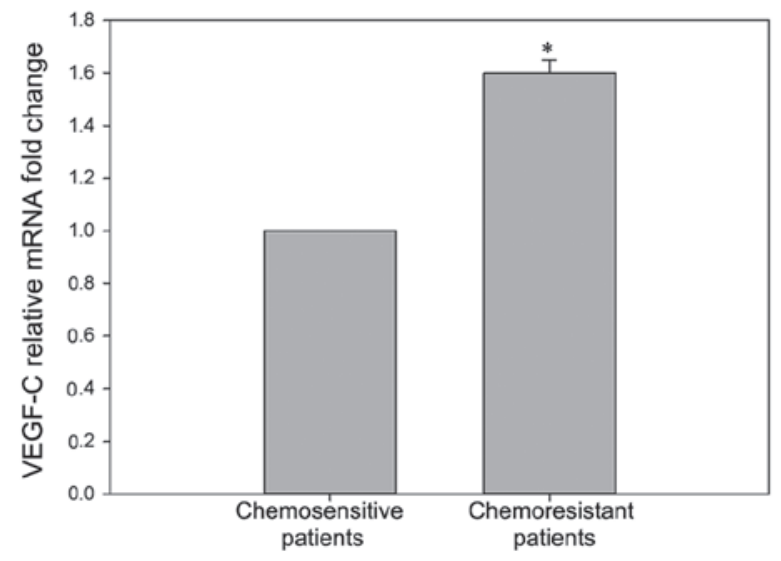

Figure 1. Relative mRNA expression of VEGF-C is significantly higher in chemoresistant patients compared with chemosensitive patients. ${ }^{*} \mathrm{P}<0.05$. VEGF-C, vascular endothelial growth factor.

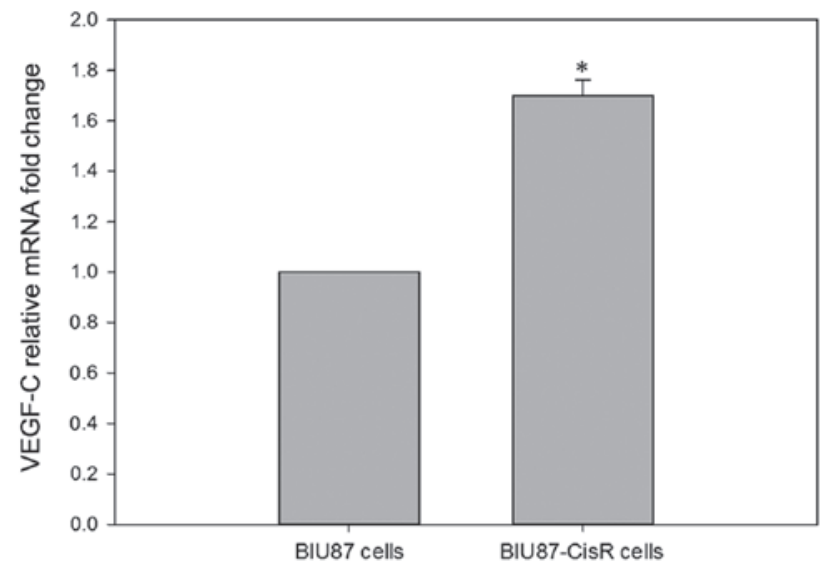

Figure 2. Relative mRNA expression of VEGF-C is significantly higher in BIU87-CisR cells compared with normal BIU87 cells. "P $<0.05$. VEGF-C, vascular endothelial growth factor $\mathrm{C}$.

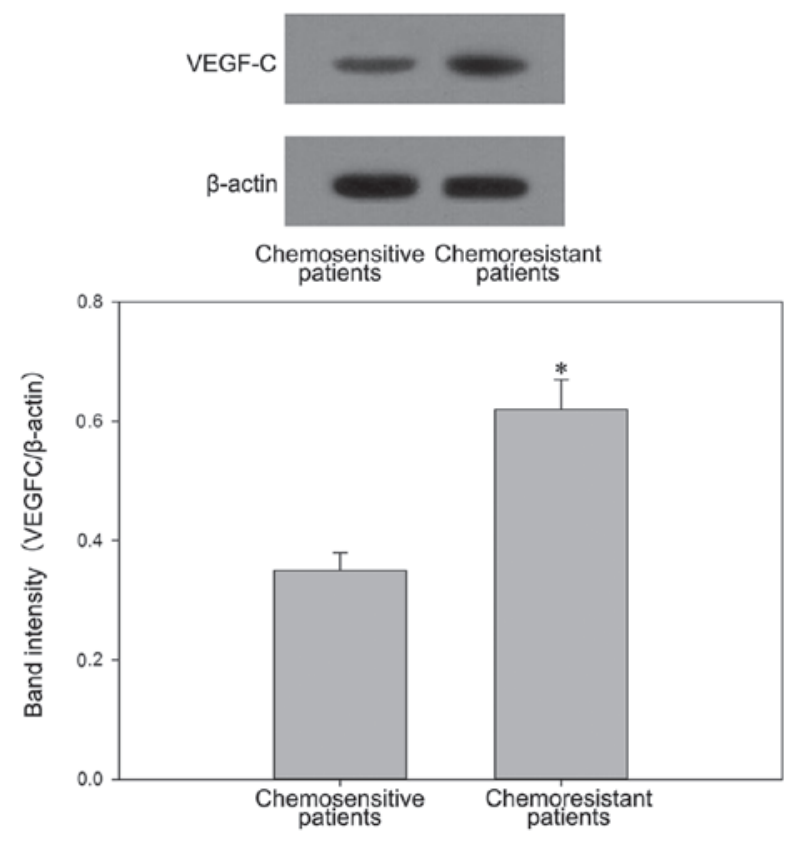

Figure 3. Western blot analysis confirms significantly higher VEGF-C protein expression in chemosenstive patients. Band intensity was normalized by $\beta$-actin. ${ }^{*} \mathrm{P}<0.05$. VEGF-C, vascular endothelial growth factor $\mathrm{C}$. 

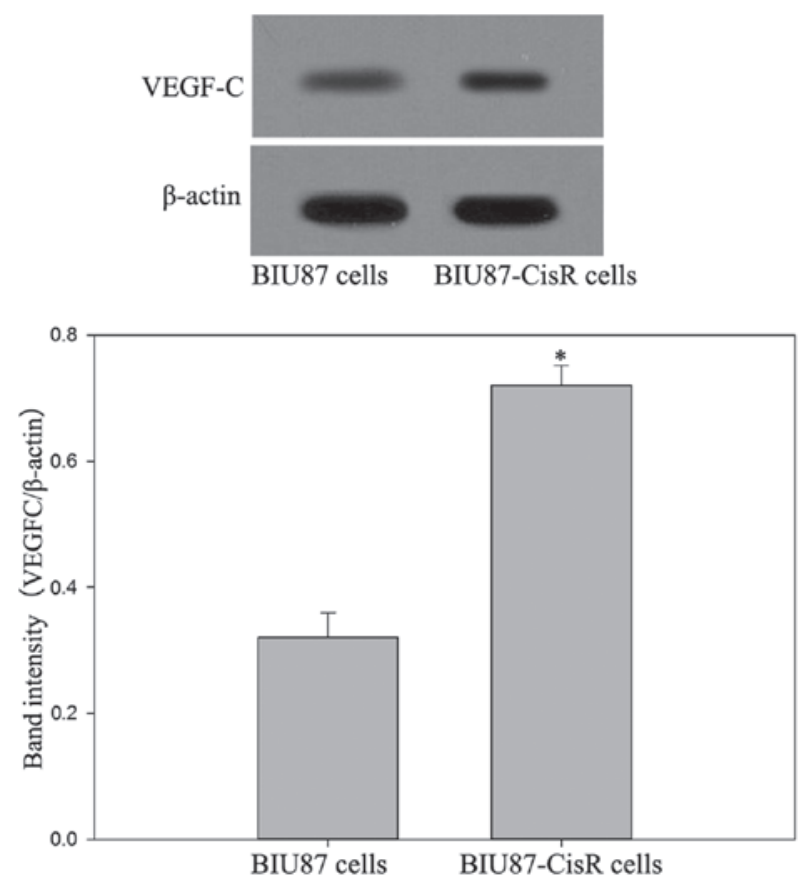

Figure 4. Western blot analysis confirms significantly higher VEGF-C protein expression in BIU87-CisR cells. Band intensity was normalized by $\beta$-actin. ${ }^{*} \mathrm{P}<0.05$. VEGF-C, vascular endothelial growth factor $\mathrm{C}$.

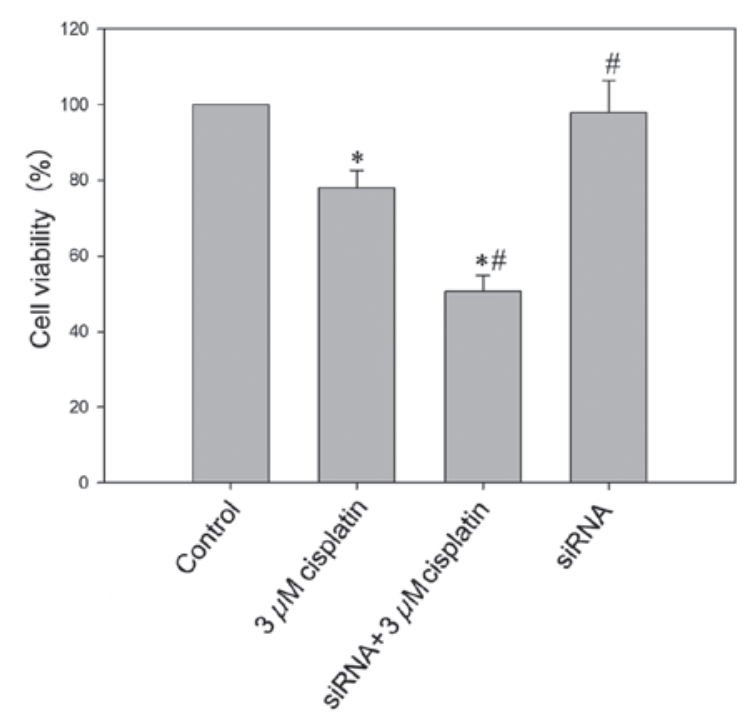

Figure 5. Cell viability in each group. Cisplatin $(3 \mu \mathrm{M})$ results in proliferation inhibition in BIU87-CisR cells while VEGF-C silencing results in further cell death. ${ }^{*} \mathrm{P}<0.05$ vs. control. ${ }^{*} \mathrm{P}<0.05$ vs. $3 \mu \mathrm{M}$ cisplatin treated group. VEGF-C, vascular endothelial growth factor $\mathrm{C}$.

$3 \mu \mathrm{M}$ cisplatin treatment may significantly induce cell death with a cell viability rate of $78.1 \pm 3.6 \%$ ( $\mathrm{P}<0.05$ vs. the control group). In VEGF-C-silenced BIU87-CisR cells, $3 \mu \mathrm{M}$ cisplatin resulted in a lower cell viability rate $(50.6 \pm 4.6 \%)$ and the difference was significant compared with other groups $(\mathrm{P}<0.05)$.

Knock-down of VEGF-C contributed to $G_{2} / M$ phase arrest of BIU87-CisR cells induced by cisplatin. Cisplatin was able to arrest BIU87-CisR cells at the $\mathrm{G}_{2} / \mathrm{M}$ phase, as presented in

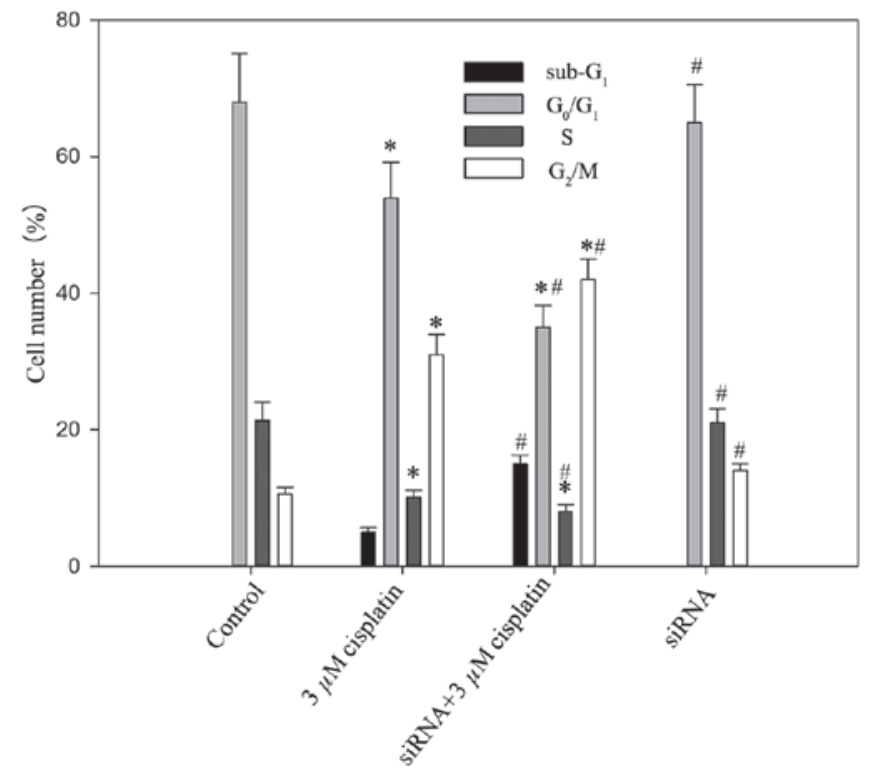

Figure 6 . Flow cytometric analysis of the cell cycle. Cisplatin $(3 \mu \mathrm{M})$ results in cell cycle arrest at $\mathrm{G}_{2} / \mathrm{M}$ phase in BIU87-CisR cells, and VEGF-C inhibition reinforces this effect. The figure shows the proportion of cells in the $G_{0} / G_{1}, S$ and $\mathrm{G}_{2} / \mathrm{M}$ phase. ${ }^{*} \mathrm{P}<0.05$ vs. control. ${ }^{\#} \mathrm{P}<0.05$ vs. $3 \mu \mathrm{M}$ cisplatin treated group.

Fig. 6. In the control group, the cell population percentages were $68.34 \pm 7.45 \%$ in the $\mathrm{G}_{0} / \mathrm{G}_{1}$ phase and $10.5 \pm 0.9 \%$ in the $\mathrm{G}_{2} / \mathrm{M}$ phase. Following treatment with $3 \mu \mathrm{M}$ cisplatin, the proportion of $\mathrm{G}_{0} / \mathrm{G}_{1}$ phase cells was reduced to $53.9 \pm 5.3 \%$, and the proportion of cells in the $\mathrm{G}_{2} / \mathrm{M}$ phase increased to $31.3 \pm 4.1 \%$. In addition, VEGF-C silencing suppressed the proportion of $\mathrm{G}_{0} / \mathrm{G}_{1}$ phase cells $(35.5 \pm 3.5 \%)$ and elevated the percentage of cells in the $\mathrm{G}_{2} / \mathrm{M}$ phase $(42.3 \pm 3.1 \%)$. The differences between each group were statistically significant $(\mathrm{P}<0.05)$.

Apoptotic rate of BIU87-CisR cells resulting from cisplatin increase due to inhibition of VEGF-C. In agreement with the results of the cell cycle analysis, the silencing of VEGF-C was observed to enhance the apoptosis-promoting effect of cisplatin. As presented in Fig. 7, which is a representative image, the apoptotic rate in the control group was $5.3 \pm 0.4 \%$, whereas following treatment with $3 \mu \mathrm{M}$ cisplatin, the apoptotic rate was increased to $17.1 \pm 3.1 \%$. For the VEGF-C-silenced BIU87-CisR cells, the apoptotic rate with cisplatin was increased to $29.3 \pm 4.5 \%$. The differences between each group were statistically significant $(\mathrm{P}<0.05)$.

Inhibition of VEGF-C increases the expression levels of maspin, which may improve the sensitivity of BIU87-CisR cells to cisplatin. In the VEGF-C-silenced BIU87-CisR cells, high mRNA and protein expression levels of maspin were observed, and a small increase in maspin expression was observed in the cisplatin-treated group compared with the control (Figs. 8 and 9). As presented in Fig. 10, following treatment with $3 \mu \mathrm{M}$ cisplatin, maspin-overexpressing BIU87-CisR cells exhibited a lower cell viability compared with normal BIU87-CisR cells $(65.1 \pm 3.9 \%$ vs. $75.3 \pm 5.6 \%$; $\mathrm{P}<0.05)$. This suggests that inhibition of VEGF-C improves the sensitivity of bladder cancer cells to cisplatin via the upregulation of maspin expression. 


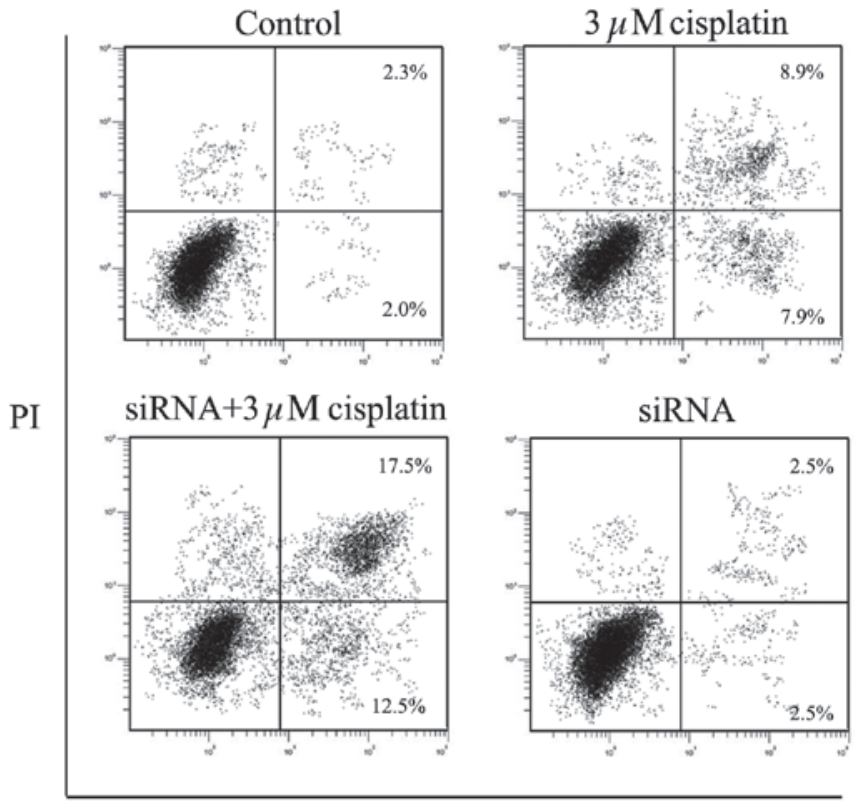

Annexin- $\mathrm{V}$

Figure 7. Flow cytometric analysis of cell apoptosis. Cisplatin $(3 \mu \mathrm{M})$ induces apoptosis of BIU87-CisR cells and VEGF-C inhibition reinforces this effect. VEGF-C, vascular endothelial growth factor C; PI, propidium iodide.

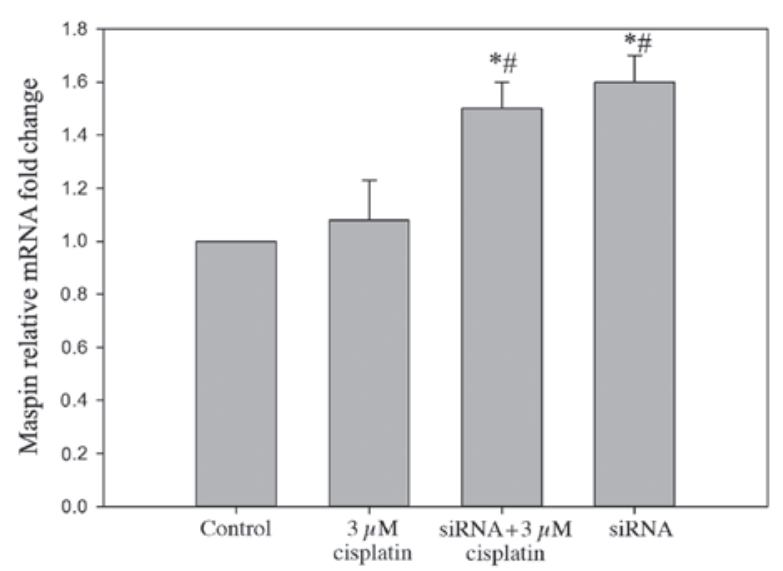

Figure 8. VEGF-C inhibition increases relative mRNA expression of maspin. ${ }^{*} \mathrm{P}<0.05$ vs. control. ${ }^{\#} \mathrm{P}<0.05$ vs. $3 \mu \mathrm{M}$ cisplatin treated group. VEGF-C, vascular endothelial growth factor C; siRNA, small interfering RNA.

\section{Discussion}

The current study supports a crucial role for VEGF-C expression in modulating the resistance of bladder cancer cells to cisplatin, VEGF-C inhibition is suggested to lead to chemosensitization through inducing maspin expression. Cisplatin is an effective broad-spectrum anticancer drug, however, extensive previous studies have reported cisplatin resistance in human cancer cells in vivo and in vitro $(12,14)$. Cisplatin sensitivity and resistance is complex and alterations can occur in almost every mechanism influencing cell growth, developmental pathways, apoptosis, DNA repair, drug metabolism and drug transporters (15). Previous studies aiming to elucidate the underlying mechanism have identified that the level of several of expression of genes contributes

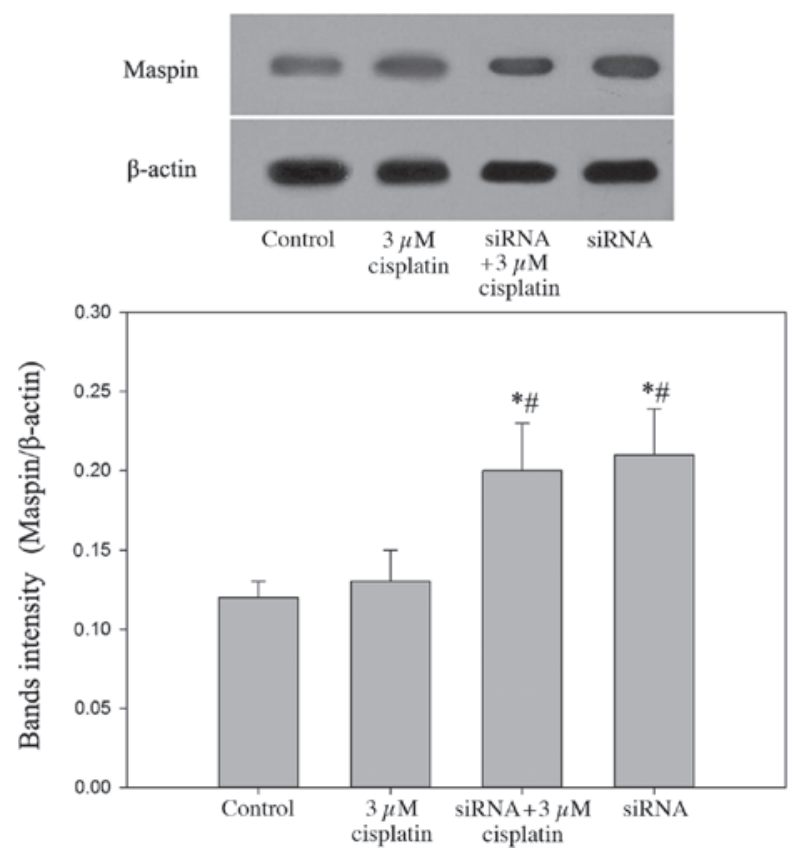

Figure 9. VEGF-C inhibition increases protein expression of maspin. Band intensity was normalized by $\beta$-actin. ${ }^{*} \mathrm{P}<0.05$ vs. control. ${ }^{\#} \mathrm{P}<0.05$ vs. $3 \mu \mathrm{M}$ cisplatin treated group. VEGF-C, vascular endothelial growth factor $\mathrm{C}$; siRNA, small interfering RNA.

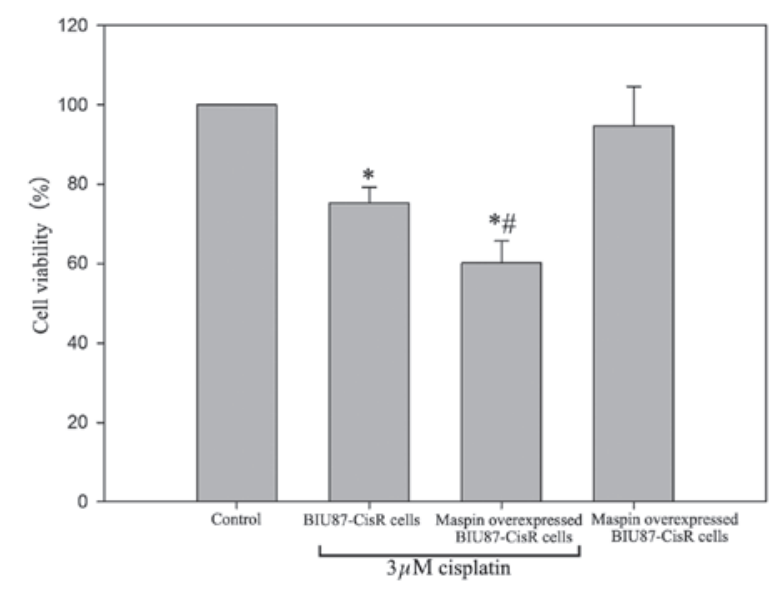

Figure 10. Maspin-overexpressing BIU87-CisR cells are more sensitive to $3 \mu \mathrm{M}$ cisplatin. ${ }^{*} \mathrm{P}<0.05$ vs. control. ${ }^{\#} \mathrm{P}<0.05$ vs. normal BIU87-CisR cells.

to the chemoresistance, and these levels are often abnormal in patients with cancer (13). These alterations in gene expression can suppress or promote tumor cell growth and apoptosis. Jayachandran et al (16) observed that the induction of NPRL2 expression by plasmid vectors containing human NPRL2 cDNA were able to overcome cisplatin resistance in non-small cell lung cancer cells. Furthermore, NPRL2 is an accepted tumor suppressor gene $(17,18)$. Hour et al $(19)$ indicated that expression of the CCAAT/enhancer binding protein $\Delta$ (CEBPD) gene was specifically elevated in a cisplatin resistant subline. CEBPD was able to antagonize reactive oxygen species and apoptosis via inducing the expression of $\mathrm{Cu} / \mathrm{Zn}$-superoxide dismutase (20). In addition to the above studies, the classical tumor suppressor gene, p53, is also important. A larger number of studies have examined 
the association between p53 and cisplatin-resistance, for example, Gutekunst et al (20) suggested that siRNA-mediated silencing of p53 abrogated hypersensitivity to cisplatin. According to this line of reasoning, it is hypothesized that any tumor suppressor or pro-oncogenic gene should be taken into consideration when investigating the resistance to cisplantin in carcinoma cells. Thus, the expression of VEGF-C was investigated due to the following reasons: As a tumor-promoter, it induces metastasis through enhancing angiogenesis, lymphangiogenesis and cancer cell invasion; the presence of high levels of VEGF-C is an accepted risk factor for poor prognosis; and its effect on promoting tumor growth has been confirmed in several studies $(21,22)$. In the current study, tissue specimens of patients with bladder cancer were analyzed, and the results indicated that expression of VEGF-C was significantly higher in chemoresistant patients compared with chemosensitive patients. In addition, high expression levels of VEGF-C were detected in the BIU87-CisR cell line, but not in the normal BIU87 cell line. Subsequent to VEGF-C inhibition, cisplatin-treated BIU87-CisR cells exhibited increased cell death, cell cycle arrest and apoptosis. These results supported the theory that high levels of VEGF-C result in resistance to cisplatin. With regard to the downstream mechanism, Hua et al (23) identified that in acute myeloid leukemic cells, VEGF-C induced cyclooxygenase-2-mediated resistance to chemotherapy through the induction of endothelin 1 expression (23). An additional study demonstrated that inhibition of the expression of VEGF-C may reverse drug resistance by regulating the activity of mTOR complex 1 (24). However, it was suggested that the expression of maspin was involved in induction of resistance to cisplatin by VEGF-C. This was hypothesized for two reasons: Firstly, maspin has been demonstrated to exhibit tumor-suppressing activities by inducing apoptosis (25), in addition it has been reported to be expressed in normal mammary epithelial cells but absent in mammary carcinoma cell lines, with a tendancy for the level of maspin to fall in line with tumor promotion and progression in humans (26). In addition, it has been previously reported that low levels of maspin maintain cancer cell growth and survival. For example, maspin is downregulated in breast and prostate cancer, which results in reduced cell motility $(11,27)$. In non-small cell lung carcinoma cells, high-maspin expressing cells were significantly less invasive and apoptotic than low-maspin expressing cells $(28,29)$. Secondly, there is an association between the expression of maspin and the chemotherapeutic response. Surowiak et al (30) demonstrated that ovarian cancer cell lines expressing maspin in the cytoplasm were more sensitive to cisplatin, and for ovarian cancer, maspin expression is associated with a longer survival rate. In the current study, the expression of maspin was detected in bladder cancer tissue and in the cisplatin-resistant BIU87 subline. The results suggest that chemoresistant patients exhibit lower levels of maspin expression when compared with chemosensitive patients. In addition, low expression levels of maspin expression were observed in the BIU87-CisR cell line. Subsequent to treatment with VEGF-C-targeted siRNA, the maspin expression levels of BIU87-CisR cells were significantly increased. Subsequently, overexpression of maspin in BIU87-CisR cells induced by a recombinant plasmid enhanced the proliferation inhibition effect of cisplatin. These results suggest that VEGF-C inhibition reverses the resistance of bladder cancer cells to cisplatin via upregulating maspin. However, there are limitations in the current study as follows: The maspin protein is regulated by methylation of the gene promoter, however, investigation of whether VEGF-C is associated with DNA methylation was not conducted. In addition, maspin expression should also be subjected to investigation as a potential surrogate marker for cisplatin sensitivity of individual patients in vivo.

In conclusion, the resistance of bladder cancer cells to cisplatin may be induced by upregulation of VEGF-C, since inhibition of VEGF-C reverses resistance by increasing the expression levels of maspin.

\section{References}

1. Xu W, Wang F, Ying L and Wang HH: Association between glutathione S-transferase M1 null variant and risk of bladder cancer in Chinese Han population. Tumour Biol 35: 773-777, 2014.

2. Costantini C and Millard F: Update on chemotherapy in the treatment of urothelial carcinoma. ScientificWorldJournal 11: 1981-1994, 2011.

3. Shirato A, Kikugawa T, Miura N, Tanji N, Takemori N, Higashiyama $\mathrm{S}$ and Yokoyama M: Cisplatin resistance by induction of aldo-keto reductase family 1 member $\mathrm{C} 2$ in human bladder cancer cells. Oncol Lett 7: 674-678, 2014.

4. Galluzzi L, Vitale I, Michels J, Brenner C, Szabadkai G, Harel-Bellan A, Castedo M and Kroemer G: Systems biology of cisplatin resistance: Past, present and future. Cell Death Dis 5: e1257, 2014.

5. Ding M, Fu X, Tan H, Wang R, Chen Z and Ding S: The effect of vascular endothelial growth factor $C$ expression in tumor-associated macrophages on lymphangiogenesis and lymphatic metastasis in breast cancer. Mol Med Rep 6: 1023-1029, 2012.

6. Ciobanu M, Eremia IA, Crăiţ̧oiu S, Mărgăritescu CL, Stepan A, Pătraşcu V, Georgescu CC, Cernea D and Dumitrescu D: Lymphatic microvessels density, VEGF-C and VEGFR-3 expression in 25 cases of breast invasive lobular carcinoma. Rom J Morphol Embryol 54: 925-934, 2013.

7. Liu J, Cheng Y, He M and Yao S: Vascular endothelial growth factor $\mathrm{C}$ enhances cervical cancer cell invasiveness via upregulation of galectin-3 protein. Gynecol Endocrinol 30: 461-465, 2014.

8. Ma DM, Xu YP and Zhu L: Expression of vascular endothelial growth factor $\mathrm{C}$ correlates with a poor prognosis based on analysis of prognostic factors in patients with cervical carcinomas. J Obstet Gynaecol Res 37: 1519-1524, 2011.

9. Cho HJ, Kim IK, Park SM, et al: VEGF-C mediates RhoGDI2-induced gastric cancer cell metastasis and cisplatin resistance. Int J Cancer 135: 1553-1563, 2014.

10. Snoeren N, Emmink BL, Koerkamp MJ, et al: Maspin is a marker for early recurrence in primary stage III and IV colorectal cancer. Br J Cancer 109: 1636-1647, 2013.

11. Machowska M, Wachowicz K, Sopel M and Rzepecki R: Nuclear location of tumor suppressor protein maspin inhibits proliferation of breast cancer cells without affecting proliferation of normal epithelial cells. BMC Cancer 14: 142, 2014.

12. Lopez-Ayllon BD, Moncho-Amor V, Abarrategi A, Ibañez de Cáceres I, Castro-Carpeño J, Belda-Iniesta C, Perona R and Sastre L: Cancer stem cells and cisplatin-resistant cells isolated from non-small-lung cancer cell lines constitute related cell populations. Cancer Med 3: 1099-1111, 2014.

13. Zhou BH, Huang JN, Zuo YL, Li BJ, Guo Q, Cui BC, Shao WY, Du J and Bu XZ: 2a, a novel curcumin analog, sensitizes cisplatin-resistant A549 cells to cisplatin by inhibiting thioredoxin reductase concomitant oxidative stress damage. Eur J Pharmacol 707: 130-139, 2013.

14. Ajani JA, Wang X, Song S, et al: ALDH-1 expression levels predict response or resistance to preoperative chemoradiation in resectable esophageal cancer patients. Mol Oncol 8: 142-149, 2014. 
15. Shen DW, Pouliot LM, Hall MD and Gottesman MM: Cisplatin resistance: a cellular self-defense mechanism resulting from multiple epigenetic and genetic changes. Pharmacol Rev 64: 706-721, 2012

16. Jayachandran G, Ueda K, Wang B, Roth JA and Ji L: NPRL2 sensitizes human non-small cell lung cancer (NSCLC) cells to cisplatin treatment by regulating key components in the DNA repair pathway. PLoS One 5: e11994, 2010.

17. Ling KS, Chen GD, Tsai HG, Lee MS, Wang PH and Liu FS: Mechanisms involved in chemoresistance in ovarian cancer. Taiwan J Obstet Gyne 44: 209-217, 2005.

18. Patel NP, Pattni BS, Abouzeid AH and Torchilin VP: Nanopreparations to overcome multidrug resistance in cancer. Adv Drug Deliver Rev 65: 1748-1762, 2013.

19. Hour TC, Lai YL, Kuan CI, et al: Transcriptional up-regulation of SOD1 by CEBPD: a potential target for cisplatin resistant human urothelial carcinoma cells. Biochem Pharmacol 80: 325-334, 2010

20. Gutekunst M, Mueller T, Weilbacher A, Dengler MA, Bedke J, Kruck S, Oren M, Aulitzky WE and van der Kuip H: Cisplatin hypersensitivity of testicular germ cell tumors is determined by high constitutive Noxa levels mediated by Oct-4. Cancer Res 73: 1460-1469, 2013.

21. Zhang J, Zhu Z, Sun Z, Sun X, Wang Z and Xu H: Survivin gene expression increases gastric cancer cell lymphatic metastasis by upregulating vascular endothelial growth factor- $\mathrm{C}$ expression levels. Mol Med Rep 9: 600-606, 2014.

22. Li D, Xie K, Ding GT, Li J, Chen K, Li H, Qian J, Jiang C and Fang J: Tumor resistance to anti-VEGF therapy through up-regulation of VEGF-C expression. Cancer Lett 346: 45-52, 2014.
23. Hua KT, Lee WJ, Yang SF, Chen CK, Hsiao M, Ku CC, Wei LH, Kuo ML and Chien MH: Vascular endothelial growth factor-C modulates proliferation and chemoresistance in acute myeloid leukemic cells through an endothelin-1-dependent induction of cyclooxygenase-2. Biochim Biophys Acta 1843: 387-397, 2014.

24. Stanton MJ, Dutta S, Zhang H, Polavaram NS, Leontovich AA, Hönscheid P, Sinicrope FA, Tindall DJ, Muders MH and Datta K: Autophagy control by the VEGF-C/NRP-2 axis in cancer and its implication for treatment resistance. Cancer Res 73: 160-171, 2013.

25. Zou Z, Anisowicz A, Hendrix MJ, Thor A, Neveu M, Sheng S, Rafidi K, Seftor E and Sager R: Maspin, a serpin with tumor-suppressing activity in human mammary epithelial cells. Science 263: 526-529, 1994

26. Taskiran C, Erdem O, Onan A, Vural C, Arisoy O, Yildiz S and Guner H: Maspin expression in endometrial hyperplasia and carcinoma and its relation with angiogenesis. Eur J Gynaecol Oncol 35: 134-139, 2014.

27. Berardi R, Morgese F, Onofri A, et al: Role of maspin in cancer. Clin Transl Med 2: 8, 2013.

28. Hirai K, Koizumi K, Haraguchi S, et al: Prognostic significance of the tumor suppressor gene maspin in non-small cell lung cancer. Ann Thorac Surg 79: 248-253, 2005.

29. Takanami I, Abiko T and Koizumi S: Expression of maspin in non-small-cell lung cancer: Correlation with clinical features. Clin Lung Cancer 9: 361-366, 2008.

30. Surowiak P, Materna V, Drag-Zalesinska M, Wojnar A, Kaplenko I, Spaczyński M, Dietel M, Zabel M and Lage H: Maspin expression is characteristic for cisplatin-sensitive ovarian cancer cells and for ovarian cancer cases of longer survival rates. Int J Gynecol Pathol 25: 131-139, 2006. 